\title{
Convective assembly of a particle monolayer
}

\author{
N A Fleck ${ }^{1,2}$, R M McMeeking ${ }^{2,3,4}$, T Kraus $^{2}$ \\ ${ }^{1}$ Corresponding author, Cambridge University Engineering Dept., Trumpington St., \\ Cambridge, CB2 1PZ, UK \\ ${ }^{2}$ INM - Leibniz Institute for New Materials, Campus D2 2, Saarbrücken, Germany \\ ${ }^{3}$ Mechanical Engineering and Materials Departments, University of California, Santa \\ Barbara, CA 93106, USA \\ ${ }^{4}$ School of Engineering, University of Aberdeen, King's College, Aberdeen, AB24 2UE, \\ UK
}

$18 / 11 / 15$

\section{Summary}

Recently, the steady-state process of convective assembly has emerged as a viable production route for colloidal monolayers. The present study models the regions of particle assembly: Region I comprises convective concentration of a particle suspension in a liquid below a meniscus, Region II comprises permeation of fluid through the dense particle monolayer, and Region III comprises capillary densification. For each region, the dominant physics and non-dimensional groups are identified and quantitative models are derived to describe the evolution of microstructure in terms of the main process parameters. The concentration profile within the assembly zone of Region I is predicted, including the role of a concentration-dependent diffusion constant and the shape of the meniscus. The fluid flow through the assembled monolayer is treated in Region II, along with a stability calculation to reveal that isolated particle clusters do not survive on top of the monolayer. The physics of capillary crystallization is addressed in Region III, with an emphasis on the density of cracks that emerge. The Peclet number and Capillary number both play important roles but in different regions of the assembly process. 


\section{Introduction}

Convective assembly is an emerging self-assembly technique for thin particle layers of dense packing and controlled crystallinity from a dilute dispersion, see Fig. 1. We consider here the steady-state deposition of a single layer on a moving substrate, with particle assembly driven by convection, capillarity, and by evaporation of the liquid. The process relies upon a stable colloidal dispersion. A colloid is a dispersion of small particles in a liquid, with the particles small enough to be subjected to Brownian motion ${ }^{1}$. For example, polymer particles (such as PS or PMMA) can exist as a stable suspension in water when stabilized by a surface charge.

The technique of convective assembly can be used to coat macroscopic areas with dense, homogeneous particle layers without the need for vacuum equipment or elevated temperatures. Applications include thin decorative layers of pigment particles, antireflective layers of refractive particles on transparent substrates, and diffusion barriers composed of non-permeable platelets. Under appropriate processing conditions, the coating has a crystalline structure making it suitable for use as an optical grating ${ }^{2,3}$. A wide range of particle size can be assembled, ranging from a few nanometers for proteins to the micron scale for polymer lattices ${ }^{4}$.

Convective assembly requires the establishment of a stable liquid meniscus to guide the flow of the suspension onto a substrate. Existing wet coating equipment can be adopted for this task. Industrial slot-die coaters, doctor blades, dip coaters and similar machinery are available for substrate widths of several meters, for liquid film thicknesses down to the micron range and for a wide range of substrate velocities up to meters per second ${ }^{5}$. The main objectives of the present study are to (i) develop the underlying theoretical basis for the convective assembly technique, (ii) give a unified treatment of all stages of deposition, and (iii) predict the required process parameters that yield high-quality particle coatings. The main contribution is to identify the essential physics dominating each stage of steady-state convective assembly by evaluating the magnitude of nondimensional groups that combine the relevant physical variables. Models are formulated and predictions are made on the basis of the dominant physics. 


\subsection{Previous work}

Drying regimes maps have been constructed for particulate coatings, see for example, Cardinal et $\mathrm{al}^{6}$. They solve a convection-diffusion equation for the transient problem of a drying film in one dimension and explore the significance of gravity by introducing a sedimentation term. Two non-dimensional groups control the distribution of particles within the film: a Peclet number Pe and a sedimentation number $\mathrm{N}_{\mathrm{G}}$. The particles are driven upwards to the free surface by solvent evaporation provided $\mathrm{Pe}>>1$ and $\mathrm{N}_{\mathrm{G}}<<1$. Sedimentation drives the particles downwards when $P e>>1$ and $N_{G}>1$. Diffusion maintains a uniform concentration of particles for $\mathrm{Pe}<<1$ regardless of the magnitude of $\mathrm{N}_{\mathrm{G}}$

The assembly mechanisms analysed below assume that the assembly process is in steadystate. This is in contrast to the transient processes of sedimentation ${ }^{6}$, filtration ${ }^{7-9}$ and spin coating $^{10,11}$. We limit the remainder of our review of the literature to steady-state particle assembly processes for which surface tension dictates the geometry of flow and for which gravity plays a negligible role.

Many of the underlying principles of colloidal assembly are summarized in the seminal monograph of Russel et $\mathrm{al}^{1}$. Based on an earlier study by Batchelor ${ }^{12}$, they emphasise the role of colloidal interactions in the diffusion of particles and provide a generalized Stokes-Einstein diffusion equation (page 430, equation 13.1.2) for a non-dilute suspension. The model presented herein builds upon this treatment. Russel and Routh have, in a long and fruitful collaboration and separately, analyzed many aspects of layer formation from colloidal dispersions involving diffusion, convection and sedimentation. For example, they have considered the evolution of particle distribution during solvent evaporation from films using a diffusion-convection equation ${ }^{13}$. Russel, Routh and coworkers have not analysed the present problem of convective assembly in steady-state.

Brewer et $\mathrm{al}^{14}$ have generated regime maps for convective assembly in steady state by a detailed experimental study. They varied the solid concentration and the substrate velocity over a wide range and observed a set of morphologies in the as-deposited state. For example, they found that a continuous monolayer gave way to an incomplete monolayer when the forced convection of a drying gas (nitrogen) was switched off or the solids contribution was reduced. The present study is complementary to their approach: 
whilst Brewer and co-workers revealed the regimes of dominance experimentally in terms of a capillary number and initial particle concentration we shall analyse in some depth the process of monolayer deposition.

Our strategy is to construct an overall model for convective assembly based upon a sequence of 3 regions as shown in Fig. 1: (I) convective accumulation of particles submerged in a liquid, (II) permeation of fluid through the dense particle layer, and (III) capillary densification. Previous studies on convective assembly have not broken the process into discrete regions, each with their own distinctive set of physical phenomena. For example, Dimitrov and Nagayama ${ }^{15}$ developed an expression for the growth velocity of the layer based on a continuity statement. Norris et al. ${ }^{16}$ considered the fluid mechanics of the assembly step, particle-by-particle, and emphasized the crystallinity of the deposited layer. Nagayama ${ }^{17}$ addressed particle assembly by a combination of convection and capillarity but neglected the role of diffusion. In contrast, an effectivemedium approach is adopted in the present study, with due attention paid to diffusion and non-dilute particle interactions.

\section{Overview of the 3 Regions of Flow}

We identify 3 main regions of flow, I to III as sketched in Fig. 1, consistent with the observations of Born et $\mathrm{al}^{18}$. Region I involves convective accumulation of particles, Region II is dominated by the permeation of fluid through the dense layer, and capillary densification occurs in the final Region III. In addition, a pre-cursor region 0 of convective assembly exists between the doctor blade and substrate, and comprises a suspension of uniform height $h_{\text {in }}$. Each Region is analysed in terms of a limited set of dimensionless groups, and these are used to identify the operating window of convective assembly. We begin by listing representative values of the main physical parameters that characterize the problem.

\subsection{Typical Process Parameters}

Colloidal convective assembly can occur over a range of process parameters. However, for illustration, it is instructive to give a typical set of parameters, similar to those in Born et $\mathrm{al}^{18}$. A colloidal suspension of spherical particles, of volume fraction $\phi_{\text {in }} \approx 10^{-3}$ and diameter $d \approx 0.5 \mu \mathrm{m}$, is supplied from an upstream reservoir of height $h_{\text {in }} \approx 1 \mathrm{~mm}$, 
supported by a moving substrate, see Fig. 1. The density, viscosity and surface tension of the dilute suspension are close to those of water, $\rho \approx 1000 \mathrm{~kg} \mathrm{~m}^{-3}, \eta \approx 10^{-3} \mathrm{~Pa}$ and $\gamma \approx$ $0.1 \mathrm{~N} \mathrm{~m}^{-1}$, respectively. The diffusion constant $D$ for the particles in the liquid is described in the dilute limit by the Stokes-Einstein relation $D=k T /(3 \pi \eta d) \approx 8.6 \times 10^{-13}$ $\mathrm{m}^{2} \mathrm{~s}^{-1}$ where $k=1.38 \times 10^{-23} \mathrm{~J} \mathrm{~K}^{-1}$ is Boltzmann's constant and $T$ is absolute temperature, see for example Batchelor ${ }^{19}$.

In Region I, the colloidal particles are transported within a converging meniscus by combined convection and diffusion. The height $h(x)$ of the meniscus reduces from $h_{\text {in }}$ to $h\left(\ell_{\mathrm{I}}\right) \approx d$ over its length $\ell_{\mathrm{I}} \approx 1 \mathrm{~mm}$. The meniscus ends with vanishing contact angle and sits on top of a fully wetted monolayer of particles.

At the end of Region I, the particle concentration attains a value $\phi_{\mathrm{d}}$ (on the order of dense packing), and the particle velocity equals that of the substrate, $v_{\text {sub }} \approx 1 \mu \mathrm{m} \mathrm{s}^{-1}$. The liquid permeates the dense particle layer in the ensuing Region II. Continuity dictates that the velocity of the liquid is significantly greater than $v_{\text {sub }}$. Evaporation of the liquid from the surface of the layer occurs at a rate $\dot{q} \approx 40 \mathrm{~nm} \mathrm{~s}^{-1}$ that depends upon the ambient temperature and humidity. Consequently, the liquid slows down in this socalled Region II of length $\ell_{\mathrm{II}} \approx 1 \mathrm{~mm}$. At the end of Region II, the liquid level drops below the top surface of the particles, and capillary forces then pull the particles together and hold them to the substrate. This Region III of capillary densification is of length $\ell_{\text {III }} \approx 10 \mu \mathrm{m}$, and drying cracks may develop within it.

\subsection{Dimensional Analysis}

It is instructive to re-express the above physical variables in non-dimensional form in order to reduce the number of independent parameters, and to identify the dominant phenomena. The list for Region I is as follows (see for example page 371 of Russel et $\left.\mathrm{al}^{1}\right)$ : 
(i) The Reynolds Number of the fluid $\operatorname{Re} \equiv \rho v_{\text {sub }} d / \eta$ gives the ratio of inertial forces on the fluid to viscous forces, and is on the order of $10^{-6}$. Hence, inertial effects of the fluid (material acceleration and turbulence) can be neglected, and flow is laminar.

(ii) The Stokes Number of the particles $\mathrm{St} \equiv\left(\rho_{\mathrm{p}} d / \rho \ell\right)$ Re gives the ratio of inertial forces on the particles to viscous forces by the fluid, where $\rho_{\mathrm{p}}$ is the density of particles and $\ell$ is a characteristic length such as the length $\ell_{\mathrm{I}}$ of Region I. Typically, the Stokes number St is on the order of $10^{-9}$, implying that the inertia of particles is negligible.

(iii) The Peclet Number $\mathrm{Pe}=d v_{\text {sub }} / D$ gives the ratio of convection to diffusive transport, and is of order unity, implying that particle transport is by a combination of convection and diffusion.

(iv) The Froude Number $\mathrm{Fr} \equiv v_{\text {sub }} / \sqrt{g d}$ gives the ratio of inertial forces to gravitational forces (as described by the acceleration due to gravity, $g$ ). We find that $\mathrm{Fr} \approx 10^{-3}$ implying that the flow is stable: hydraulic jumps and shallow water waves are absent.

(v) The Sedimentation Number $\mathrm{N}_{\mathrm{G}} \equiv d^{2} \Delta \rho g /\left(\mu v_{\text {sub }}\right)$ is a function of the density difference $\Delta \rho$ between particles and liquid, and gives the ratio of sedimentation velocity to the substrate velocity. It is of order $10^{-2}$, confirming that sedimentation due to gravity is negligible.

(vi) The Capillary Number $\mathrm{Ca} \equiv \eta v_{\text {sub }} / \gamma$ gives the ratio of viscous forces to surface tension. It is of order $10^{-8}$, implying that surface tension sets the meniscus shape.

We conclude from the above values of non-dimensional groups that convection and diffusion dominate particle transport within the curved meniscus of Region I. The effects of particle sedimentation and inertia are negligible. This motivates the convectiondiffusion analysis of the following section.

In Region II, the particles are submerged just below the flat meniscus, and the curvature of the liquid is negligible. Since the Capillary Number $\mathrm{Ca} \equiv \eta v_{\text {sub }} / \gamma$ has the same small value as in Region I, we conclude that the flow of liquid within Region II exerts negligible viscous forces on the meniscus so that it remains flat throughout. 
In contrast, the particles are partially submerged in Region III, and the small value of Capillary Number implies that capillary forces drive the densification of particles, with a negligible role played by viscosity and diffusion.

\section{Region I: convective assembly}

A dilute suspension of particles, of diameter $d$, forms a meniscus of profile $h(x)$ above a substrate moving at $v_{\text {sub }}$, recall Fig. 1. The meniscus is of parabolic shape and of length $\ell_{\mathrm{I}}$ approximately equal to the capillary length

$$
\kappa^{-1}=\sqrt{\gamma / \rho g}
$$

see for example section 2.3 of de Gennes et al. ${ }^{20}$. Particles assemble into a layer of height $d$ by convection, but assembly is impeded by diffusion. Region I ends where the particles have attained the velocity $v_{\text {sub }}$; in the ensuing Region II the meniscus is of height $h=d$ and the liquid moves at a velocity $v_{1}>>v_{\text {sub }}$. We shall simplify the analysis within Regions I and II by assuming that the velocity of particles and liquid do not vary over the height of the layer.

\subsection{Continuity}

We now identify a further stage of convection-diffusion to the left of Region I. This region is of uniform thickness between the substrate and the doctor blade, and is termed Region 0 . At the inlet to Region 0 the liquid suspension has a height $h_{\text {in }}$ and a volume fraction of particles $\phi_{\text {in }}$. At this location, the particles and liquid share the velocity $v_{\text {in }}$, and the particle flux over the height $h_{\text {in }}$ is $J_{\mathrm{s}}=\phi_{\mathrm{in}} v_{\mathrm{in}} h_{\text {in }}$. Similarly, the liquid flux at entry to Region 0 is $J_{1}=\left(1-\phi_{\text {in }}\right) v_{\text {in }} h_{\text {in }}$. Within Regions 0 and I the particles exhibit Brownian motion with an ensemble average velocity $\left\langle v_{\mathrm{s}}\right\rangle(x)$ due to convection and diffusion and the particle flux times the height is given by $J_{\mathrm{S}}=\phi\left\langle v_{\mathrm{s}}\right\rangle h$. Likewise, the fluid has an ensemble average velocity $\left\langle v_{1}\right\rangle(x)$, giving rise to a fluid flux $J_{1}=(1-\phi)\left\langle v_{1}\right\rangle h$. Volume conservation in Regions 0 and I requires

$$
J_{\mathrm{s}}=\phi\left\langle v_{\mathrm{s}}\right\rangle h=\phi_{\text {in }} v_{\text {in }} h_{\text {in }}
$$


and

$$
J_{1}=(1-\phi)\left\langle v_{1}\right\rangle h=\left(1-\phi_{\text {in }}\right) v_{\text {in }} h_{\text {in }}
$$

Addition of these two fluxes gives

$$
J=J_{\mathrm{s}}+J_{1}=v_{\mathrm{av}} h=v_{\text {in }} h_{\text {in }}
$$

where the average velocity of the suspension at any location $x$ is

$$
v_{\mathrm{av}}=\phi\left\langle v_{\mathrm{s}}\right\rangle+(1-\phi)\left\langle v_{1}\right\rangle
$$

In Region 0 the film height and average velocity are both uniform. As flow proceeds in the direction of increasing $x$ in Region I, the height of the film thickness $h(x)$ drops while the average suspension velocity increases. The volume fraction $\phi(x)$ of particles rises to a value $\phi_{\mathrm{d}}$ at the end of Region I. It is convenient to locate the origin of the co-ordinate $x$ at the end of Region I, as shown in Fig. 1.

\subsection{Convection-diffusion model}

The flux of particles $J_{\mathrm{s}}$ across any representative section of height $h(x)$ is driven in the forward $x$-direction by convection and driven backwards by diffusion down the concentration gradient $\partial \phi / \partial x$ such that

$$
J_{\mathrm{s}}=\phi v_{\mathrm{av}} h-D \frac{\partial \phi}{\partial x} h=\phi_{\mathrm{in}} v_{\mathrm{in}} h_{\mathrm{in}}
$$

This can be rearranged to non-dimensional form by making use of (3.3) to read

$$
\frac{\phi}{\phi_{\text {in }}}-\frac{1}{\mathrm{Pe}_{\text {in }}} \frac{D}{D_{\text {in }}} \frac{h}{h_{\text {in }}} \frac{\partial\left(\phi / \phi_{\text {in }}\right)}{\partial(x / d)}=1
$$

where the inlet Peclet number $\mathrm{Pe}_{\text {in }}$ is defined by

$$
\mathrm{Pe}_{\text {in }}=\frac{d v_{\text {in }}}{D_{\text {in }}}
$$

At exit from Region I, the particles form a layer of height $d$ and attain the substrate velocity $v_{\text {sub }}$, such that $J_{\mathrm{s}}=\phi_{\mathrm{d}} v_{\text {sub }} d$. Upon equating this flux to the inlet value (as stated on the r.h.s. of (3.5)) we obtain an expression for the Peclet number in terms of $v_{\text {sub }}$ rather than in terms of $v_{\text {in }}$ : 


$$
\mathrm{Pe}_{\text {in }}=\frac{\phi_{\mathrm{d}} v_{\mathrm{sub}} d^{2}}{\phi_{\text {in }} h_{\text {in }} D_{\text {in }}}
$$

We shall argue below that the exit value $\phi_{\mathrm{d}}$ at $x=0$ is a function of the Peclet number, and its value provides the required boundary condition for the solution of the convectiondiffusion equation (3.6). However, the precise relation between $\phi_{\mathrm{d}}$ and $\mathrm{Pe}$ awaits experimental determination; a first attempt at a theoretical model is given in section (3.4) below.

\subsection{Non-linear convection-diffusion in a curved meniscus at constant diffusivity}

Equation (3.6) allows for the possibility of a shaped profile $h(x)$ and a diffusion coefficient that depends upon $\phi$. For the present problem (both at large and at small $\mathrm{Ca}$ ), the meniscus is a circular arc that can be approximated by a quadratic profile of curvature $\kappa$ and a minimum height $d$ such that

$$
h=d+\frac{1}{2} \kappa x^{2}
$$

where $\kappa=\sqrt{\rho g / \gamma}$ from (3.1). An analytical solution exists for the case where $D$ is assumed constant throughout Regions 0 and I.

First consider Region 0. In this case Eq. (3.6) simplifies to

$$
\frac{\phi}{\phi_{\text {in }}}-\frac{1}{\mathrm{Pe}_{\text {in }}} \frac{\partial\left(\phi / \phi_{\text {in }}\right)}{\partial(x / d)}=1
$$

The solution to Eq. (3.9a) is

$$
\phi(x)=\phi_{\text {in }}+\left(\phi_{0}-\phi_{\text {in }}\right) \exp \left[\frac{\mathrm{Pe}_{\text {in }}\left(x+l_{\mathrm{I}}\right)}{d}\right]
$$

where $\phi_{0}=\phi\left(-l_{\mathrm{I}}\right)$, i.e. the value of $\phi$ where the suspension enters Region I. Straightforward integration of (3.6) for Region I then gives

$$
\phi(x)=\phi_{\text {in }}+\left(\phi_{\mathrm{d}}-\phi_{\text {in }}\right) \exp \left(\operatorname{Pe}_{\text {in }} \frac{h_{\text {in }}}{d}\left(\frac{2}{\kappa d}\right)^{1 / 2} \tan ^{-1}\left(\left(\frac{\kappa d}{2}\right)^{1 / 2} \frac{x}{d}\right)\right)
$$

and thus 


$$
\phi_{0}=\phi_{\text {in }}+\left(\phi_{\mathrm{d}}-\phi_{\text {in }}\right) \exp \left(-\mathrm{Pe}_{\text {in }} \frac{h_{\text {in }}}{d}\left(\frac{2}{\kappa d}\right)^{1 / 2} \tan ^{-1}\left(\left(\frac{\kappa d}{2}\right)^{1 / 2} \frac{l_{\mathrm{I}}}{d}\right)\right)
$$

For the limiting case of $\kappa$ approaching zero Eq. (3.10) reduces to

$$
\phi(x)=\phi_{\text {in }}+\left(\phi_{\mathrm{d}}-\phi_{\text {in }}\right) \exp \left(\operatorname{Pe}_{\text {in }} \frac{h_{\text {in }}}{d} \frac{x}{d}\right)
$$

with $h_{\text {in }} / d$ only slightly greater than unity. We note from (3.11) that the particle volume fraction increases from its initial value $\phi_{\text {in }}$ to $\phi_{\mathrm{d}}$ over a characteristic length $\left(h_{\text {in }} \mathrm{Pe}_{\text {in }}\right)^{-1} d^{2}=\left(h_{\text {in }} v_{\text {in }}\right)^{-1} D_{\text {in }} d$. In reality, this underestimates the characteristic length due to particle-particle interactions within the concentrated suspension near the exit of Region I. To address this, a full numerical solution is needed.

\subsection{Non-linear convection-diffusion in a curved meniscus and variable diffusivity}

The generalized Stokes-Einstein equation for $D$ is of the form

$$
D(\phi)=D_{0} K(\phi) \frac{d}{d \phi}[\phi Z(\phi)]
$$

in terms of a compressibility factor $Z(\phi)$ and a sedimentation coefficient $K(\phi)$, as discussed by Davis and Russel ${ }^{8}$ and more recently by Routh and Zimmerman ${ }^{13}$. Here, we follow Russel et $\mathrm{al}^{1}$ and use a hard-sphere simulation such that

$$
Z(\phi)=\frac{1.85}{\phi_{c}-\phi}
$$

where $\phi_{c}$ is a limiting packing density such as 0.64 for dense random packing. Davis and Russel $^{8}$ performed a curve fit to Buscall's sedimentation data for polystyrene particles $^{21}$ to obtain

$$
K(\phi)=(1-\phi)^{6.55}
$$

The sedimentation coefficient $K$ corrects for the effect of a concentrated solution upon Stokes sedimentation.

Recent experiments by Born et $\mathrm{al}^{18}$ indicate that the packing density $\phi_{\mathrm{d}}$ at the end of Region I depends upon the Peclet number. An approximate scaling argument can be used 
to give additional insight. Consider the forces on a representative particle at the end of Region I: the particle is subjected to thermal agitation and to viscous drag. Consider first thermal agitation, treat the particles as an ideal gas, and assume that they possess an average thermal energy $k T$. The 'gas pressure' $p$ of the particles is of order

$$
p \approx \frac{\phi_{\mathrm{d}} \mathrm{k} T}{d^{3}}
$$

Second, assume that this pressure counteracts the viscous force/unit area involved in particle assembly,

$$
p \approx \frac{\eta\left(v_{1}-v_{\mathrm{sub}}\right)}{d}
$$

where $v_{1}$ is the velocity of the liquid passing between the particles, which move at the velocity $v_{\text {sub }}$. Now make use of the Stokes-Einstein equation $D=k T /(3 \pi \eta d)$, and assume that $\left(v_{1}-v_{\text {sub }}\right)$ scales with $v_{\text {sub }}$. Upon equating (3.15) and (3.16) we obtain

$$
\phi_{\mathrm{d}}=\frac{\left(v_{1}-v_{\mathrm{sub}}\right) \eta d^{2}}{k T} \approx \frac{v_{\mathrm{sub}} d}{D}=\mathrm{Pe}
$$

Relation (3.17) suggests that particle packing density $\phi_{\mathrm{d}}$ is linear in the Peclet number but the functional dependence awaits experimental validation: in broad terms, it is expected that a large Peclet number leads to denser packing of the assembled particles, with relatively little free volume present for random motion of each particle relative to its neighbours. Born et $\mathrm{al}^{18}$ support this by direct observation of particle motion during assembly. They find that $\phi_{\mathrm{d}}$ increases with an increase in Pe. Also, Mittal et al ${ }^{22}$ observe that needle-like titania particles align in a film at high Peclet numbers, whereas the film is isotropic and the particles are randomly arranged when Brownian motion dominates at low Peclet numbers.

\subsection{Numerical solution of the non-linear convection-diffusion equation}

We proceed to solve (3.6) by making use of (3.12)-(3.14). Introduce the non-dimensional groups $\tilde{x} \equiv x / d, \tilde{\phi} \equiv \phi / \phi_{\text {in }}, \tilde{h}=h / h_{\text {in }}$ and $\tilde{D} \equiv D / D_{\text {in }}$ to obtain

$$
\tilde{\phi}-\frac{\tilde{D} \tilde{h}}{\mathrm{Pe}_{\text {in }}} \frac{\partial \tilde{\phi}}{\partial \tilde{x}}=1
$$


The end of Region I is located at $\tilde{x}=0$, and the boundary condition there reads $\tilde{\phi}=\tilde{\phi}_{\mathrm{d}} \equiv \phi_{\mathrm{d}} / \phi_{\text {in }}$. Rearrangement of (3.18) leads to

$$
\int_{\tilde{\phi}}^{\tilde{\phi}_{\mathrm{d}}} \frac{\tilde{D}\left(\phi^{\prime}\right)}{\phi^{\prime}-1} d \phi^{\prime}=\mathrm{Pe}_{\text {in }} \int_{\tilde{x}}^{0} \frac{1}{\tilde{h}\left(x^{\prime}\right)} d x^{\prime}=\tilde{\mathrm{P}} \int_{\tilde{x}}^{0}\left(1+\frac{1}{2} \kappa x^{\prime 2}\right) d x^{\prime}
$$

where $\tilde{\mathrm{P}} \equiv v_{\text {in }} h_{\text {in }} / D_{\text {in }}=\mathrm{Pe}_{\text {in }} h_{\text {in }} / d$.

Newton iteration and numerical integration gives $\tilde{\phi}(\tilde{x})$ for selected values of $\tilde{\mathrm{P}}$ and $\tilde{\phi}_{\mathrm{d}}$. Results are shown in Fig. 2 for selected values of $\phi_{d}$, for the choice $\phi_{\text {in }}=0.001$, $\phi_{\mathrm{c}}=0.64$, and $\kappa d=1.6 \times 10^{-4}$. Provided $\tilde{\mathrm{P}}$ exceeds a value of about 0.3 , the zone of convective assembly is short compared to the capillary length $\kappa^{-1}$ and the curvature of the meniscus can be neglected. The results in Fig. 2 are plotted as solid curves for the choice $\tilde{\mathrm{P}}=10$, with an additional plot (dotted line) given for $\tilde{\mathrm{P}}=0.1$ and $\phi_{\mathrm{d}}=0.639$; there is only a minor change in response when $\tilde{\mathrm{P}}$ is decreased from 10 to 0.1 for this choice of $\phi_{\mathrm{d}}$. Results for $\tilde{\mathrm{P}}=0.1$ and $\phi_{\mathrm{d}}=0.6,0.4$ and 0.2 are omitted as the results overlap (to the line width) with those for $\tilde{\mathrm{P}}=10$. It is clear that the characteristic length over which $\tilde{\phi}(\tilde{x})$ increases from the inlet to outlet value increases as $\tilde{\phi}_{\mathrm{d}}$ approaches $\tilde{\phi}_{\mathrm{c}}$ consistent with the feature that $D \rightarrow \infty$ as $\phi_{\mathrm{d}} \rightarrow \phi_{\mathrm{c}}$. We note that the inlet condition $\phi=\phi_{\text {in }}$ at the entrance to Region I is not satisfied exactly in the numerical results; this feature is also present in the special case given in Eq. (3.10). However, Fig. 2 makes it clear that the discrepancy is negligible.

It is instructive to give the asymptotic behaviour for $\tilde{\phi}(\tilde{x})$ near the end of Region I. Assume that $\phi_{\mathrm{d}}$ is slightly less than $\phi_{\mathrm{c}}$ and rewrite $\phi$ as

$$
\phi=\phi_{\mathrm{c}}-\Delta \phi
$$

Then, at $x=0$, we have $\Delta \phi_{\mathrm{d}} \equiv \phi_{\mathrm{c}}-\phi_{\mathrm{d}}$. The expression for $D$ from (3.12)-(3.14) becomes

$$
D \approx \frac{A}{(\Delta \tilde{\phi})^{2}} D_{0}
$$


where $A=1.85\left(1-\phi_{\mathrm{c}}\right)^{6.55} \phi_{\mathrm{c}} /\left(\phi_{\text {in }}\right)^{2}$. Substitution of (3.20) and (3.21) into (3.6), and reexpression in non-dimensional form gives to leading order

$$
\frac{\partial \Delta \tilde{\phi}}{\partial \tilde{x}}=\frac{\operatorname{Pe}_{\text {in }}\left(1-\tilde{\phi}_{\mathrm{c}}\right)}{A \tilde{h}(\Delta \tilde{\phi})^{2}}
$$

with solution

$$
(\Delta \tilde{\phi})^{-1}=\left(\Delta \tilde{\phi}_{\mathrm{d}}\right)^{-1}-\frac{\tilde{\mathrm{P}}\left(1-\tilde{\phi}_{\mathrm{c}}\right)}{A}\left(\frac{2}{\kappa d}\right)^{1 / 2} \tan ^{-1}\left(\left(\frac{\kappa d}{2}\right)^{1 / 2} \tilde{x}\right)
$$

The small final slope of $\phi$ at the end of Region I follows immediately as

$$
\frac{\partial \tilde{\phi}}{\partial \tilde{x}}=\tilde{\mathrm{P}} \frac{\left(\tilde{\phi}_{\mathrm{c}}-1\right)\left(\Delta \tilde{\phi}_{\mathrm{d}}\right)^{2}}{A}
$$

We conclude from the full solution (3.19) or from the asymptotic result (3.24) that the particle concentration builds up over a rather narrow zone of length $d / \tilde{\mathrm{P}}$. Recent experiments suggest that this prediction could be verified by measuring the particle concentration in situ: Goehring and co-workers analyzed the X-ray scattering during evaporation and report particle volume fractions, for example $\mathrm{Li}$ et $\mathrm{al}^{23}$.

\section{Region II: fluid permeation through the layer}

In Region II, the particles lie just below the surface of the liquid, and liquid evaporates from the surface at a flux rate of $\dot{q}$ per unit area. The liquid surface is flat by the following qualitative argument. Recall from section 2 that the Reynolds number and Capillary number are both small, implying that inertial forces are much less than viscous forces, and in turn viscous forces are much less than capillary forces. Consequently, a change in liquid velocity can occur with negligible pressure gradients arising from viscosity or inertia, and the surface of the liquid remains flat.

The above qualitative argument can be made quantitative by assuming that viscous Darcy flow though the particle packing is driven by the pressure drop $\Delta p_{\mathrm{m}}$ associated with a gas/liquid interface of curvature $\kappa_{\mathrm{m}}$ as discussed by Mason and Mellor ${ }^{24}$, and Russel ${ }^{25}$. Idealise the Darcy flow as Poiseuille flow through a cylindrical tube of diameter $d$ and length $\ell$. Then, the pressure drop $\Delta p_{\mathrm{v}}$ along the tube reads 


$$
\Delta p_{\mathrm{v}} \approx \frac{\eta v_{1} \ell}{d^{2}}
$$

where $v_{1}$ is the velocity of the liquid. Upon equating this to the pressure across a curved gas/liquid interface,

$$
\Delta p_{\mathrm{m}} \approx \gamma \kappa_{\mathrm{m}}
$$

we obtain

$$
\kappa_{\mathrm{m}} d \approx \frac{\eta v_{1} \ell}{\gamma d}
$$

Now assume a fast liquid flow with $\eta=10^{-3} \mathrm{~Pa} \mathrm{~s}, v_{1}=10 \mathrm{~mm} \mathrm{~s}^{-1}, \ell=0.1 \mathrm{~mm}, d=0.5 \mu \mathrm{m}$, and $\gamma=0.1 \mathrm{Nm}^{-1}$ to obtain $\kappa_{\mathrm{m}} d \approx 0.02$. Consequently, the average meniscus curvature $\kappa_{\mathrm{m}}$ is much less than $1 / d$. This result can be re-phrased in terms of Capillary number $\mathrm{Ca} \equiv \eta v_{\text {sub }} / \gamma$ as follows. The expression $\Delta p_{\mathrm{V}}<<\Delta p_{\mathrm{m}}$ gives via the identities (4.2) and (4.3),

$$
\mathrm{Ca} \ll 5 \frac{d}{\ell} \frac{v_{\mathrm{sub}}}{v_{1}}=2.5 \times 10^{-3}
$$

and this is fulfilled in any practical deposition process, as noted by Brewer et $\mathrm{al}^{14}$. In summary, inviscid flow occurs through the particle packing and the pressure gradient in the forward $x$-direction is negligible. Note that the maximum liquid velocities in the particle packing far surpass the liquid velocities in the meniscus of Region I.

To first order (until more detailed experimental data become available), we take $\dot{q}$ to be constant within Region II (and within Region III). Continuity of liquid volume within Region II implies that the liquid decelerates until it attains the average particle velocity $v_{\text {sub }}$. Evaporation of the liquid slows down the liquid until $v_{1}=v_{\text {sub }}$. Consequently, the length of Region II is

$$
\frac{\ell_{\mathrm{II}}}{d} \approx \frac{\left(1-\phi_{\mathrm{d}}\right)\left(v_{1}-v_{\mathrm{sub}}\right)}{\dot{q}}
$$

Now substitute for typical values from section 2.1 and from Born et $\mathrm{al}^{18}$ : $\ell_{\mathrm{II}} / d=2000$, $\phi_{\mathrm{d}}=0.5, v_{1}=150 \mu \mathrm{m} \mathrm{s}^{-1}$ to obtain $\dot{q}=40 \mathrm{~nm} \mathrm{~s}^{-1}$. This estimate of evaporation rate is plausible, see for example Born et $\mathrm{al}^{18}$. During this evaporative step, surface tension 
keeps the monolayer stable in height. It is instructive to conduct a perturbation analysis in order to demonstrate its stability, as follows.

\subsection{Stability of layer thickness in Region II}

It is instructive to investigate the stability of the monolayer in Region II. Consider a perturbation in the form of a conical bump on top of the flat monolayer: the surface energy of the cone on the layer exceeds that of the flat layer alone, and this causes the cone to flatten by viscous flow within the cone. We seek a solution to this boundary value problem.

Assume that the cone has an initial height $h_{0}$ and initial radius $R_{0}$, such that the initial apex semi-angle $\alpha_{0}$ is given by $\tan \alpha_{0}=R_{0} / h_{0}$. At any time $t$, the height of the cone is $h_{b}$, its base radius is $R_{b}$ and the apex semi-angle $\alpha$ evolves to $\tan \alpha=R_{b} / h_{b}$, as defined in the sketch in Fig. 3. This is an idealization to the true evolution of shape, but it is adequate for our purposes as it captures the progressive flattening of the bump, and provides a particularly simple relation between evolution of shape and of strain field, as detailed below. Conservation of mass of the cone dictates that its volume is given by

$$
V_{b}=\frac{\pi}{3} R_{b}^{2} h_{b}=\frac{\pi}{3} R_{0}^{2} h_{0}
$$

and the area of its curved surface reads

$$
S_{b}=\frac{\pi R_{b}^{2}}{\sin \alpha}
$$

At any instant, the Helmholtz free energy $H$ of the cone can be written in terms of the surface energy $\gamma_{s}$ according to

$$
H\left(h_{b}\right)=\left(S_{b}-\pi R_{b}^{2}\right) \gamma_{s}
$$

and note that (4.6) - (4.8) allow $H$ to be stated in terms of the single degree of freedom $h_{b}$ or equivalently as a function of $\alpha$.

Now treat the wet particles as a linear viscous medium, endowed with a viscosity $\eta_{f}$, such that in uniaxial tension the axial stress $\sigma$ is related to the axial strain rate $\dot{\varepsilon}$ 
according to $\sigma=3 \eta_{f} \dot{\varepsilon}$. The dissipation rate per unit volume is $\psi=\frac{3}{2} \eta_{f} \dot{\varepsilon}^{2}$. The deformation mode of the cone is one of axial shortening, as follows. Picture the cone as embedded within a circular cylinder and sharing the same base and height. (The volume of the cone is $1 / 3$ that of the circumscribing circular cylinder). Then, as the cylinder shortens in uniaxial compression, so does the cone. The axial strain rate is $\dot{\varepsilon}=-\dot{h} / h$ and the radial strain rate equals $-\dot{\varepsilon} / 2$ by incompressibility. The dissipation potential for the cone is the volume integral of the dissipation rate per unit volume $\psi$, such that

$$
\Psi\left(\dot{h}_{b}\right)=V_{b} \psi=\frac{3 V_{b} \eta_{f} \dot{h}_{b}^{2}}{2 h_{b}^{2}}
$$

The variational method of $\mathrm{Suo}^{26}$, $\mathrm{Cocks}^{27}$ and Cocks et $\mathrm{al}^{28}$ is used to obtain an expression for the evolution of microstructure. For a given geometry, the rate of evolution of microstructure is given by the kinetic rate $\dot{h}_{b}$ that minimizes the functional

$$
\Omega\left(\dot{h}_{b}\right)=\dot{H}\left(\dot{h}_{b}\right)+\Psi\left(\dot{h}_{b}\right)
$$

such that $\delta \Omega=0$ by taking variations with respect to $\dot{h}_{b}$. Evaluation of $\dot{H}(\dot{h})$ via (4.6)(4.8) gives

$$
\dot{H}\left(\dot{h}_{b}\right)=\frac{\pi \gamma R_{0}^{2} h_{0}}{h^{2}}\left(\frac{1.5 \cos ^{2} \alpha+\sin \alpha-1}{\sin \alpha}\right) \dot{h}_{b}
$$

and evaluation of $\delta \Omega=0$ gives

$$
\dot{\alpha}=\frac{3}{2} \frac{\gamma}{\eta h_{0}}\left(\frac{\tan \alpha}{\tan \alpha_{0}}\right)^{2 / 3}\left(\frac{3}{2} \cos ^{2} \alpha+\sin \alpha-1\right) \cos \alpha
$$

Numerical quadrature of (4.12) is used to obtain $\alpha(t)$ and thence $h(t)$ via

$$
\frac{h(t)}{h_{0}}=\left(\frac{\tan \alpha_{0}}{\tan \alpha}\right)^{2 / 3}
$$

and the response $h(t)$ is plotted in Fig. 3 for selected values of $\alpha_{0}$ and $\tau \equiv \gamma /\left(\eta h_{0}\right)$. Note that the relaxation time $\tau$ of the imperfection back to a flat profile is of order $100 \tau \approx 10^{-6} \mathrm{~s}$ upon assuming the values of parameters cited in section (2.1). We conclude that the monolayer is highly stable against perturbations in thickness in Region II. The above analysis explains the observation that single layers and multi-layers form in a stable manner, whereas isolated clusters of particles are unstable during deposition. 
However, the above stability analysis does not predict why a monolayer forms in preference to multilayers. The recent experiments by Brewer et al ${ }^{14}$ reveal that multilayers form at high values of initial particle volume fraction and at high Capillary number. The onset of multilayer formation requires a refinement of the modeling as given in the current study.

\section{Region III: capillary densification}

Continued evaporation causes the level of liquid to fall below $d$. A straightforward estimate can be made for the length $\ell_{\text {III }}$ of Region III by equating the total flux of evaporated liquid $\dot{q} \ell_{\mathrm{III}}$ to the incoming flux of liquid $\left(1-\phi_{\mathrm{d}}\right) v_{\mathrm{sub}} d$ at entry to Region III, giving

$$
\frac{\ell_{\mathrm{III}}}{d} \approx \frac{\left(1-\phi_{\mathrm{d}}\right) v_{\mathrm{sub}}}{\dot{q}}
$$

Upon substituting typical values from section 2.1 we obtain $\ell_{\text {III }} / d \approx 10$. We conclude that this Region Is much shorter than the preceding ones.

Within Region III, the gas-liquid interface drapes around the top of the particles. Denkov et $\mathrm{al}^{29,30}$ observed that the transition from a disordered to ordered (crystalline) state appears suddenly when the particle tops protrude from the liquid film. Any nonuniformity of particle packing induces attractive forces between the particles and leads to capillary densification, as discussed by Kralchevsky and Nagayama ${ }^{31,32}$ and by Kralchevsky and Denkov ${ }^{33}$. As evaporation continues, the particle contacts evolve into isolated capillary bridges, and the particles are again pulled together. The justification for this perspective is as follows.

Kralchevsky and co-workers ${ }^{31,34,35}$ have extensively explored capillary interactions between particles at gas-liquid interfaces, including the case where particles rest on a solid substrate and are partially immersed by a liquid. A non-uniform distribution of particles leads to the generation of immersion forces, driven by wetting. The immersion forces much exceed the Brownian forces associated with $\mathrm{k} T$, see for example Fig. 2 of Kralchevsky and Nagayama ${ }^{31}$. Consequently, diffusion can be neglected in Region III. 
But what sets the cluster size? Consider a cracked film, formed from an initial state of mobile particles of packing density $\phi_{c}$. Crystalline clusters of uniform packing density $\phi_{f}$ form, and the clusters are separated by cracks of width on the order of the particle diameter $d$. Consider a representative circular cluster of radius $R_{c}$. Conservation of mass of solid implies that

$$
\pi\left(R_{c}+\frac{d}{2}\right)^{2} \phi_{c}=\pi R_{c}^{2} \phi_{f}
$$

and consequently

$$
\frac{2 R_{c}}{d}=\frac{\tilde{\phi}+\sqrt{\tilde{\phi}}}{1-\tilde{\phi}}
$$

where $\tilde{\phi}=\phi_{c} / \phi_{f} \leq 1 . \quad$ For example, if $\phi_{c}=0.5$ and $\phi_{f}=0.8$ then we obtain $2 R_{c} / d=4$. This is comparable to the values observed by Born et $\mathrm{al}^{18}$. Note that cluster sizes are very sensitive to the final packing, and that local perturbations may exist that we do not consider here. A full treatment would consider distributions of particle spacing but is out of our scope here.

An alternative view of the condensation processes in Region III is to assume that that phase separation occurs into a dense clusters (one phase) and voids (second phase). The pattern of clusters is reminiscent of spinodal decomposition, for which models such as the Cahn-Hilliard theory are commonly invoked, Cahn and Hilliard ${ }^{36}$. However, the underlying physics do not support this modeling approach for the following reasons:

(i) Cahn-Hilliard assumes an effective medium of continuous phases, with a continuous spatial distribution of solute (particle) concentration. Here, the packing density jumps over the length scale of the outermost particle of the cluster.

(ii) As demonstrated by Kralchevsky and $\mathrm{Nagayama}^{32}$, the capillary forces dominate Brownian forces, and consequently particles are rearranged by capillary action and not diffusion.

\section{Concluding discussion}


In the present study, the concentration profile within the assembly zone of Region I is predicted, including the role of a concentration-dependent diffusion constant and the shape of the meniscus. The fluid flow through the assembled monolayer is treated in Region II, along with a stability calculation to reveal the propensity for formation of isolated particle clusters on top of the monolayer. And, the physics of capillary crystallization is addressed in Region III, with an emphasis on the density of cracks that emerge.

The present study is a first step in the generation of a process map for convective assembly: suitable axes might be the Peclet number and $\dot{q} / v_{s u b}$. For example, the magnitude of $\dot{q} / v_{\text {sub }}$ sets the length of regions II and III, and whether a monolayer or multi-layers form ${ }^{14}$. Such a map will include the limits of operation of the above mechanisms of convective assembly. The non-dimensional groups defined in section 2.2 indicate several physical limits to the map.

The convection-diffusion model for Region I reveals a gap in our current understanding of what sets the final packing density at the end of Region I. In the current study, we suggest that this is sensitive to the Peclet number, as supported by the observations of Born et $\mathrm{al}^{18}$. But the precise functional form of $\phi_{d}(\mathrm{Pe})$ is unclear, and demands further attention, experimentally or theoretically. To date, a pragmatic approach has been adopted, whereby the diffusivity diverges near close packing by the introduction of a compressibility factor based upon a hard sphere equation of state, see for example Davis and Russel $^{8}$. In reality, repulsive interactions become important at lower packing densities than the close packed value, and the final packing density is sensitive to the Peclet number. The final packing density $\phi_{f}$ within each cluster at the end of Region III almost attains the crystalline limit, but the average number of particles within each cluster depends upon the Peclet number in accordance with relation (5.3). The theoretical treatment of the present study highlights the role of the Peclet and Capillary numbers in dictating the final film structure, and allows for an interpretation of recent experimental data $^{6,13,14,18}$.

\section{Acknowledgements}


Part of this work was performed during Norman Fleck's stay at INM that was supported by the Alexander von Humboldt Foundation. The authors acknowledge Eduard Arzt's continuing support of this project. 


\section{List of symbols}

$\begin{array}{ll}A & \text { Diffusion correction factor } \\ \mathrm{Ca} & \text { Capillary Number }\end{array}$

d Particle diameter m

$D \quad$ Particle diffusion constant $\quad \mathrm{m}^{2} \mathrm{~s}^{-1}$

$D_{0} \quad$ Particle diffusion constant at infinite dilution $\quad \mathrm{m}^{2} \mathrm{~s}^{-1}$

$D_{\text {in }} \quad$ Particle diffusion constant at inlet $\mathrm{m}^{2} \mathrm{~s}^{-1}$

Fr Froude Number -

$g \quad$ Gravitational acceleration $\mathrm{m} \mathrm{s}^{-2}$

$h \quad$ Meniscus height at position $x \quad \mathrm{~m}$

$H \quad$ Helmholtz free energy of cone J

$h_{0}$ Initial height of cone m

$h_{\mathrm{b}} \quad$ Height of cone at given time m

$h_{\text {in }}$ Meniscus height at inlet m

$J_{1} \quad$ Liquid flux over full height $\mathrm{m}^{2} \mathrm{~s}^{-1}$

$J_{\mathrm{s}} \quad$ Particle flux over full height $\mathrm{m}^{2} \mathrm{~s}^{-1}$

$\mathrm{k} \quad$ Boltzmann's constant $\mathrm{JK}^{-1}$

K Sedimentation coefficient -

l Characteristic length m

$l_{\mathrm{I}}$ Length of Region I m

$l_{\text {II }}$ Length of Region II m

$l_{\text {III }}$ Length of Region III m

$\mathrm{N}_{\mathrm{G}} \quad$ Sedimentation number -

$p \quad$ Analogous pressure exerted by particles $\quad \mathrm{Pa}$

Pe Peclet number

$\mathrm{Pe}_{\text {in }} \quad$ Peclet Number at inlet

$\dot{q} \quad$ Liquid evaporation rate $\mathrm{m} \mathrm{s}^{-1}$

$R_{0} \quad$ Initial radius of cone $\mathrm{m}$

$R_{\mathrm{b}} \quad$ Radius of cone at given time $\quad \mathrm{m}$

$R_{\mathrm{c}} \quad$ Typical particle cluster radius $\quad \mathrm{m}$

Re Reynolds Number -

$S_{\mathrm{b}} \quad$ Surface area of cone at given time $\quad \mathrm{m} 2$

St Stokes Number -

$V_{\mathrm{b}} \quad$ Volume of cone at given time m3

$v_{\text {in }} \quad$ Average velocity at inlet $\mathrm{m} \mathrm{s}^{-1}$

$v_{\mathrm{l}} \quad$ Liquid velocity $\mathrm{m} \mathrm{s}^{-1}$

$v_{\mathrm{s}} \quad$ Particle velocity $\mathrm{m} \mathrm{s}^{-1}$

$v_{\text {sub }} \quad$ Substrate velocity $\mathrm{m} \mathrm{s}^{-1}$

$x \quad$ Position on substrate m

Z Compressibility factor -

$\alpha_{0} \quad$ Initial apex semi-angle of cone $\quad$

$\alpha_{\mathrm{b}} \quad$ Apex semi-angle of cone at given time

$\gamma \quad$ Liquid surface tension $\quad \mathrm{N} \mathrm{m}^{-1}$

$\gamma_{\mathrm{s}} \quad$ Surface energy of cone J m-2

$\delta \quad$ Axial stress $\quad \mathrm{Pa}$

$\Delta p_{\mathrm{m}} \quad$ Liquid pressure drop due to capillarity $\quad \mathrm{Pa}$

$\Delta p_{\mathrm{m}} \quad$ Liquid pressure drop due to friction in pipe flow $\quad \mathrm{Pa}$

$\Delta \rho \quad$ Density difference between particle and liquid $\quad \mathrm{kg} \mathrm{m}^{-3}$ 
$\Delta \Phi_{\text {c }} \quad$ Difference to critical particle volume fraction

$\Delta \Phi_{\mathrm{d}} \quad$ Difference to dense particle volume fraction

$\dot{\varepsilon} \quad$ Axial strain rate

$\mathrm{S}^{-1}$

$\eta \quad$ Liquid viscosity

Pa s

$\eta_{\mathrm{f}} \quad$ Equivalent viscosity of wet particles

Pa s

$\kappa^{-1} \quad$ Capillary length

$\mathrm{m}$

$\kappa_{\mathrm{m}} \quad$ Curvature of gas-liquid interface in Region II

$\mathrm{m}^{-1}$

$\rho \quad$ Liquid density

$\mathrm{kg} \mathrm{m}^{-3}$

$\rho$ P Density of particles

$\mathrm{kg} \mathrm{m}^{-3}$

$\Phi \quad$ Particle volume fraction

$\Phi_{\mathrm{c}} \quad$ Limiting particle volume fraction

$\Phi_{\mathrm{d}} \quad$ Particle volume fraction at the end of Region I

$\Phi_{\mathrm{f}} \quad$ Particle cluster packing density

$\Phi_{\text {in }} \quad$ Particle volume fraction at inlet

$\psi \quad$ Dissipation rate per unit volume

$\Psi \quad$ Dissipation rate

$\mathrm{J} \mathrm{m}^{-3} \mathrm{~s}^{-1}$

$\Omega \quad$ Minimization functional

$\mathrm{J} \mathrm{s}^{-1}$

$\mathrm{J} \mathrm{s}^{-1}$

Note: Non-dimensional versions of symbols are marked with a tilde on top of the original symbol. 


\section{References}

(1) Russel, W.B.; Saville, D.A.; Schowalter, W.R. Colloidal Dispersions, Cambridge University Press, 1989.

(2) García-Santamaría, F.; Galisteo-López, J.F.; Braun, P.V.; López, C. Optical diffraction and high-energy features in three-dimensional photonic crystals. Phys. Rev. B 2005, 71, 195112.

(3) Goldenberg, L.M.; Wagner, J.; Stumpe, J.; Paulke, B.-R.; Gornitz, E. Ordered Arrays of Large Latex Particles Organized by Vertical Deposition. Langmuir 2002, 18, 3319-3323.

(4) Yuan, Z.; Petsev, D. N.; Prevo, B. G.; Velev, O. D.; Atanassov, P. Two-dimensional nanoparticle arrays derived from ferritin monolayers. Langmuir 2007, 23, 5498-5504.

(5) Schweizer, P. M.; Kistler, S. F. (Eds.): Liquid Film Coating: Scientific principles and their technological implications. London, Weinheim, etc.: Chapman \& Hall, 1997.

(6) Cardinal, C.M.; Jung, Y.D.; Ahn, K.H.; Francis, L. F. Drying Regime Maps for Particulate Coatings. AIChE journal 2010, 56, 2769-2780.

(7) Kynch, G.J. A theory of sedimentation. Trans. Faraday Soc. 1952, 48, 166-176.

(8) Davis, K. E.; Russel, W. B. An asymptotic description of transient settling and ultrafiltration of colloidal dispersions. Physics of Fluids A 1989, 1, 82-100.

(9) Lee, Y.; Clark M. M. Modeling of flux decline during crossflow ultrafiltration of colloidal suspensions. J. Membrane S. 1998, 149, 181-202.

(10) Rehg, T. J.; Higgins, B. G. Spin coating of colloidal suspensions. AIChE Journal 1992, $38,489-501$.

(11) Zhang, X. P.; Sun, B. Q.; Friend, R. H.; Guo, H. C.; Nau, D.; Giessen, H. Metallic photonic crystals based on solution-processible gold nanoparticles. Nano Lett. 2006, 6, 651-655.

(12) Batchelor, G. K. Brownian diffusion of particles with hydrodynamic interactions. $J$. Fluid Mech. 1976, 74, 1-29.

(13) Routh, A. F.; Zimmerman, W.B.. Distribution of particles during solvent evaporation from films. Chemical Eng. Sci. 2004, 59, 2961-2968.

(14) Brewer, D. D.; Shibuta T.; Francis, L.; Kumar, S.; Tsapatsis, M. Coating Process Regimes in Particulate Film Production by Forced-Convection-Assisted Drag-Out. Langmuir 2011, 27, 11660-11670.

(15) Dimitrov, A.S.; Nagayama, K. Continuous convective assembling of fine particles into two-dimensional arrays on solid surfaces. Langmuir 1996, 12, 1303-1311.

(16) Norris, D. J.; Arlinghaus, E. G.; Meng, L. L.; Heiny, R.; Scriven, L. E. Opaline photonic crystals: How does self-assembly work? Adv. Mat. 2004, 16, 1393-1399.

(17) Nagayama, K. Two-dimensional self-assembly of colloids in thin liquid films. Colloids and Surfaces A 1996, 109, 363-374.

(18) Born, P.; Munoz, A.; Cavelius, C.; Kraus, T. Crystallization mechanisms in convective particle assembly. Langmuir 2012, 28, 8300-8308.

(19) Batchelor, G. K. An introduction to fluid dynamics. Cambridge University Press, 1967. 
(20) De Gennes, P.-G.; Brochard-Wyart, F.; Quere, D. Capillarity and Wetting Phenomena, Springer, 2004.

(21) Buscall, R.; Goodwin, J. W.; Ottewill, R. H.; Tadros, Th. F. The settling of particles through Newtonian and non-Newtonian media. J. Colloid Interface Sci. 1982, 85, 78-86.

(22) Mittal, M.; Niles, R.K.; Furst, E.M. Flow-directed assembly of nanostructured thin films from suspensions of anisotropic titania particles. Nanoscale 2010, 2, 2237-2243.

(23) Li, J. Q.; Cabane, B.; Sztucki, M.; Gummel, J.; Goehring, L. Drying Dip-Coated Colloidal Films, Langmuir 2012, 28, 200-208.

(24) Mason, G.; Mellor, D. W. Simulation of Drainage and Imbibitions in a Random Packing of Equal Spheres, Journal of Colloid and Interface Science 1995, 176, 214-225.

(25) Russel, W.B. Mechanics of drying colloidal dispersions: Fluid/solid transitions, skinning, crystallization, cracking, and peeling. AIChE journal 2011, 57, 1378-1385.

(26) Suo, Z. Motions of microscopic surfaces in materials. Adv. Appl. Mech. 1997, 33, 193-294.

(27) Cocks, A. C. F. The structure of constitutive laws for the sintering of fine-grained materials. Acta Metall. 1994, 42, 2191-2210.

(28) Cocks, A. C. F., Gill, S. P. A.; Pan, J. Modelling of microstructural evolution. Advances in Applied Mechanics 1999, 36, 82-162.

(29) Denkov, N. D.; Velev, O. D.; Kralchevsky, P. A.; Ivanov, I. B.; Yoshimura, H.; Nagayama, K. Mechanism of formation of two-dimensional crystals from latex particles on substrates, Langmuir 1992, 8, 3183-3190.

(30) Denkov, N. D.; Velev, O. D.; Kralchevsky, P. A.; Ivanov, I. B.; Yoshimura, H.; Nagayama, K. Two-dimensional crystallization. Nature 1993, 361, 26.

(31) Kralchevsky, P. A.; Nagayama, K. Capillary forces between colloidal particles. Langmuir 1994, 10, 23-36.

(32) Kralchevsky, P. A.; Nagayama, K. Capillary interactions between particles bound to interfaces, liquid films and biomembranes. Advances in colloid and interface science 2000, $85,145-192$.

(33) Kralchevsky, P. A.; Denkov N. D. Capillary forces and structuring in layers of colloid particles. Current Opinion in Colloid \& Interface Science 2001, 6, 383-401.

(34) Kralchevsky, P. A.; Paunov, V. N.; Ivanov, I. B.; Nagayama, K. Capillary meniscus interaction between colloidal particles attached to a liquid-fluid interface, Journal of Colloid and Interface Science 1992, 151, 79-94.

(35) Kralchevsky, P. A.; Paunov, V. N.; Denkov, N. D.; Ivanov, I. B.; Nagayama, K. Energetical and force approaches to the capillary interactions between particles attached to a liquid-fluid interface, Journal of colloid and interface science 1993, 155, 420-437.

(36) Cahn, J.W. and Hilliard, J.E. Free energy of a nonuniform system. I. Interfacial free energy, J. Chem. Phys 1958, 28, 258. 


\section{Figure Captions}

Fig. 1. The 4 regions of flow in convective particle assembly.

Fig. 2. Evolution of concentration within Region I for the choice $\phi_{\text {in }}=0.001, \phi_{\mathrm{c}}=0.64$, $\kappa d=1.6 \times 10^{-4}$, and $\tilde{P}=v_{\text {in }} h_{\text {in }} / D_{\text {in }}=P e_{\text {in }} h_{\text {in }} / d=10$. Selected curves are also shown for $\tilde{P}=0.10$

Fig. 3. Evolution in height of a perturbation in layer thickness within Region II. 


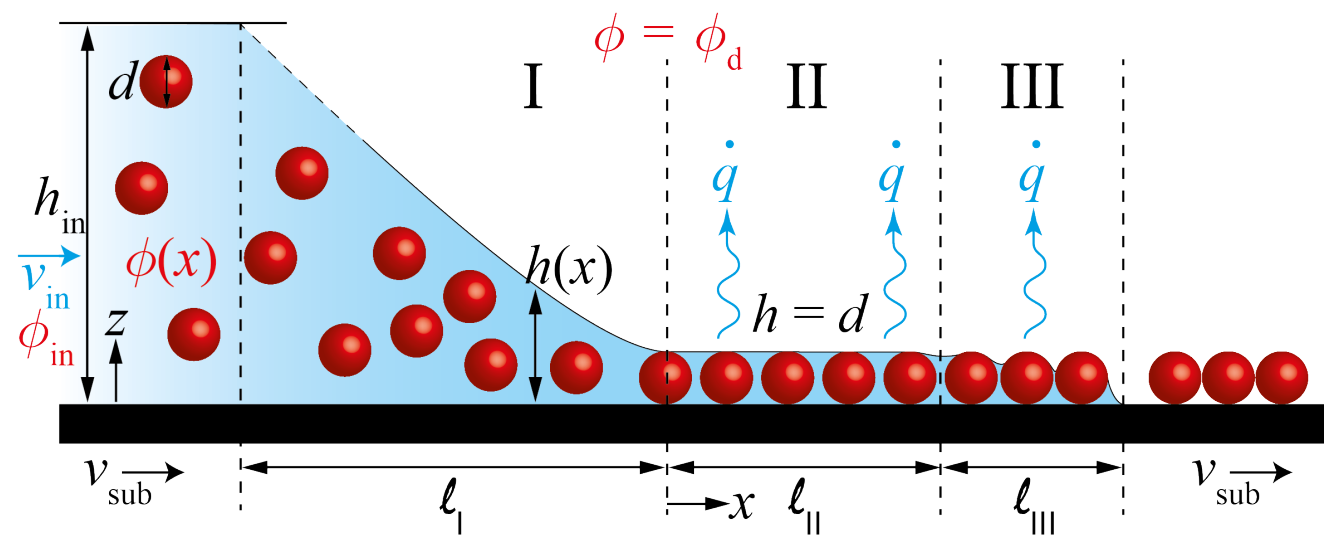

Fig. 1. The 4 regions of flow in convective particle assembly. 


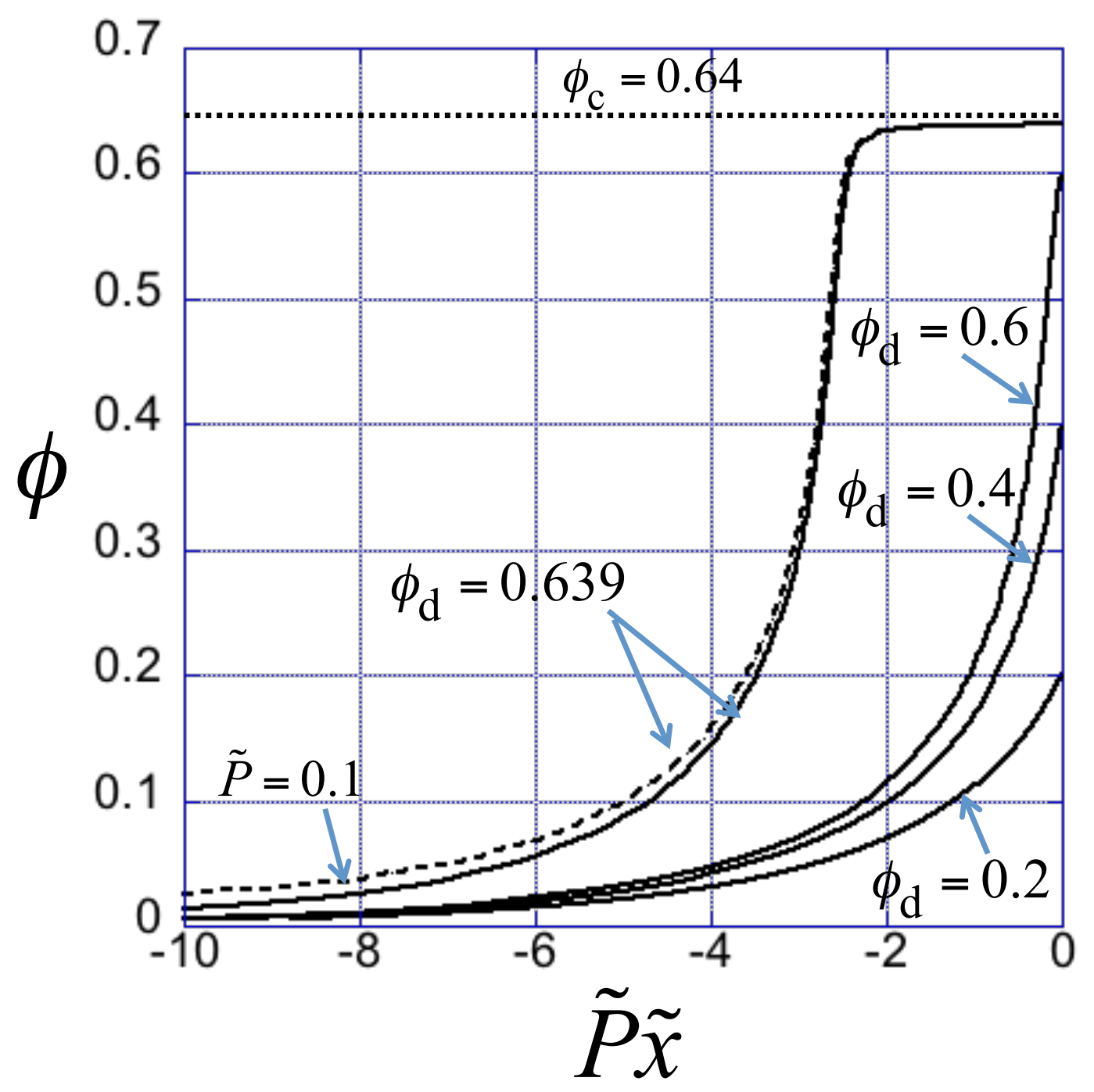

Fig. 2. The evolution of concentration within Region I for the choice $\phi_{\text {in }}=0.001$, $\phi_{\mathrm{c}}=0.64, \kappa d=1.6 \times 10^{-4}$, and $\tilde{P}=v_{\text {in }} h_{\text {in }} / D_{\text {in }}=P e_{\text {in }} h_{\text {in }} / d=10$. Selected curves are also shown for $\tilde{P}=0.10$. 


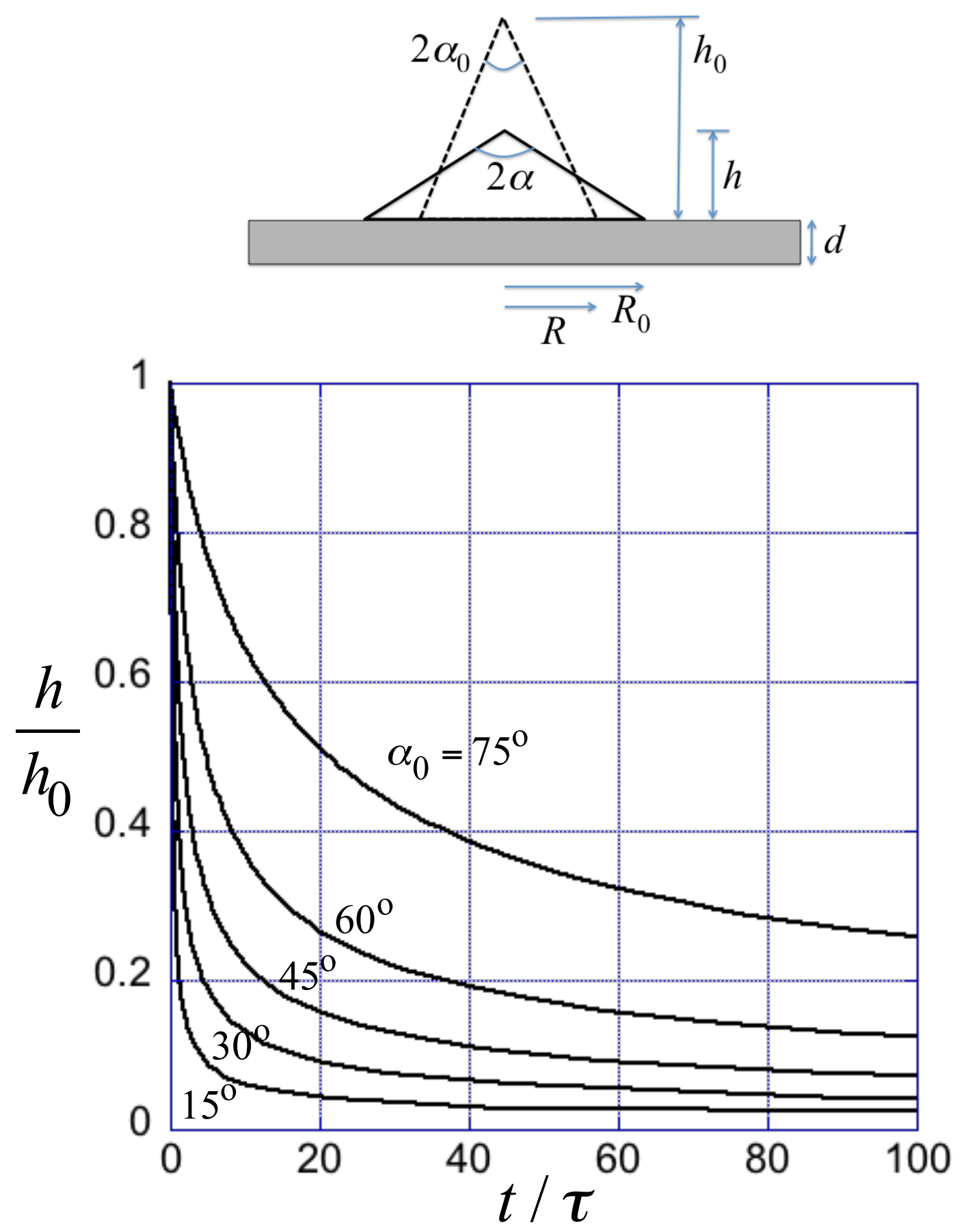

Fig. 3. Evolution in height of a perturbation in layer thickness within Region II. 
TOC Figure

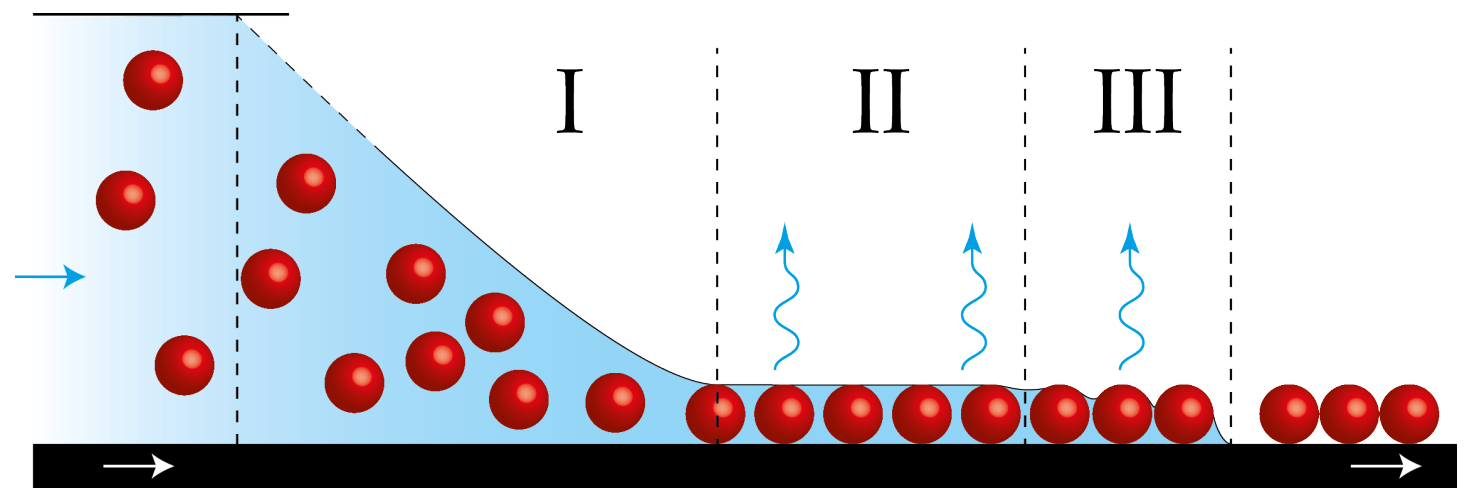

